\title{
Application of model reference adaptive control based on LS_SVM inverse model in nonlinear system
}

\author{
Shengbo Zhang, Qingling Dai \\ Jiujiang University Jiujiang, Jiangxi, 332005, China \\ E-mail: B761127@tom.com
}

Keywords: inverse model control; LS-SVM; model reference adaptive control; adaptive law

\begin{abstract}
In order to obtain better static and dynamic performance of the nonlinear system, in this paper the compound control system which the inner loop adopted direct inverse model control and the outer loop adopted model reference adaptive control was designed. Aiming at the difficulty to building inverse model in the inverse model control system in inner loop, the modeling method of inverse model based on the LS_SVM is proposed. As seen from the simulation results that the actual output signal is in good agreement with the desired output. It is show that the model reference adaptive control system based on LS_SVM inverse model has a good ability to control nonlinear object with high tracking precision, good dynamic performance and better control effect
\end{abstract}

\section{Introduction}

Recently inverse model control is treated as one of the major theoretical approaches to the study of the nonlinear control system. The basic idea of inverse model control is making use of the controlled object model to solve the equation of inverse system. And then use it to compensate the controlled object, which making the composite system to be linear system or indirect linear system on a large scale. The composite system is generally referred to the pseudo linear composite system[1]. So the practical and stable controller could be designed adopting the all kinds of the theory in linear system. But direct inverse model control is a kind of open loop control structure, there is no way to eliminate static error and lack of robustness and anti-interference ability.

The principle of model reference adaptive control is to design reference model according to the structure of the controlled object and the specific control performance requirements. In each control period the output of the reference model directly subtract the controlled object to get the generalized error[2]. Adaptive mechanism generates the self-adaptive control law using the generalized error to modify the adjustable parameters of the controller according to a certain rules and makes the error tends to zero which has strong robustness and anti-interference ability. For linear or slow time-varying system, model reference adaptive control has strong control performance. But for the nonlinear time-varying systems it is difficult to achieve the expected control effect. If combined with the both advantage and took the pseudo linear composite system consisted by controlled object and its inverse system as as the object of the model reference adaptive control system, it is a possibility to realize the optimal control of nonlinear systems .

\section{Inverse model based on LS_SVM}

For a nonlinear system, consider the following $\alpha$ order delay single input single output (SISO) system.

$$
y(k+\alpha)=f[y(k+\alpha-1), \cdots y(k+\alpha-n), u(k), \cdots, u(k-m)]
$$

Where $y(k), u(k)$ are respectively for output and input of the system. $m$ is the input delay. $n$ is the output delay. $\alpha$ is the system total delay of output to input. Taking $\alpha=1$ as an example. If the system is reversible, the reversible system can be expressed as

$$
u(k)=g[y(k+\alpha), \cdots, y(k+a-n), u(k-1), \cdots, u(k-m)]
$$

Make the input of reversible systems be $\varphi(k)=y(k+\alpha)$.Eq(2) can be expressed as 


$$
u(k)=g[\varphi(k), \cdots, \varphi(k-n), \cdots, u(k-m)]
$$

Put the inverse systems before the original system and constitutes pseudo linear compound system.

The main task of identification modeling is to determine the specific form $g(\cdot)$ and its associate parameter values.[3]. The identification modeling structure based on the inverse model of LS_SVM control system is shown as Fig 1. $u$ is the identification signal of control system. $y$ is the output signal of control system. $\hat{u}$ is the output of inverse model of LS_SVM identification. TDL is a multiple separate time delay system[4].

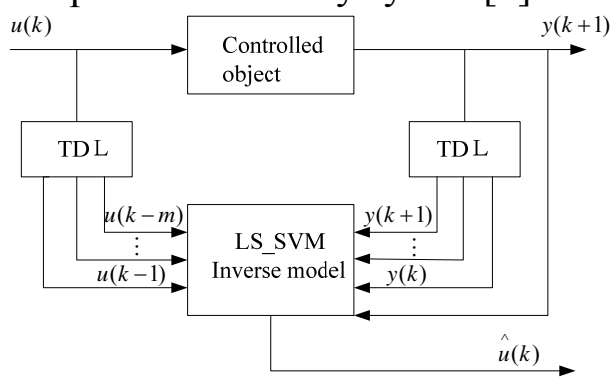

Fig. 1 the inverse model identification modeling structure

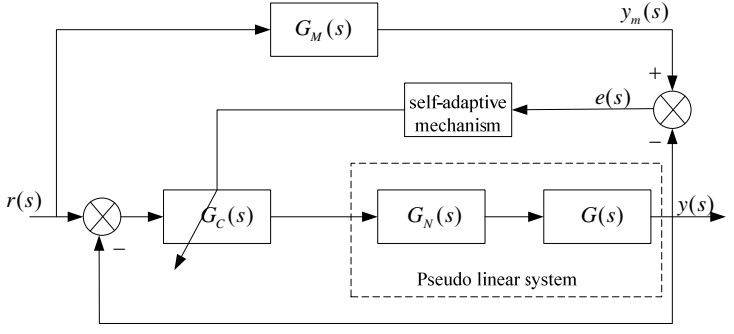

Fig.2 the compound control structure diagram

The output of the inverse model based on LS_SVM identification

$$
\hat{u}(k)=g[y(k+1), \cdots, y(k+1-n), u(k-1), \cdots, u(k-m)]
$$

Before setting up the inverse model based on LS_SVM, it wants to make sure that the training sample firstly. Assume that the input and output data sets of control system are $\{u(k), y(k), k=1,2, \cdots, N\}$ Constructed the LS_SVM training sample sets:

$$
\begin{aligned}
& D=\{(X(k-m), Y(k-m), k=(m+1), \cdots,(N-1)\} \\
& X(k-m)=\{y(k+1), \cdots, y(k+1-n), u(k-1), \cdots, u(k-m)\} \\
& Y(k-m)=u(k)
\end{aligned}
$$

The dimensions of the $X$ is $(N-m-1) \times(m+n+1), Y$ is $(N-m-1) \times 1$.After Training set $D$ was determined, LS_SVM could to proceed offline training[5] .

\section{Model reference adaptive control based on LS_SVM inverse model}

A pseudo linear system was formed by the trained inverse model series before the original system. The structure diagram is shown in Fig2. $G_{M}(s)$ is reference model; $G_{C}(s)$ is adjustable controller; $G_{N}(s)$ Is the inverse model; $G(s)$ is control object; $r(s)$ is the system input; $y(s)$ is output of control object; $e(s)$ is generalized error; $y_{m}(s)$ is output of reference model.

The core problem of the model reference adaptive control system is how to determine the adaptive control law which making the generalized error tends to zero and getting a stable system. Here using Lyapunov second method to convert the problem of adaptive control law to the stability problem. Deriveing the adaptive law of model reference adaptive control system to ensure that the system has the global asymptotic stability and better dynamic performance.

In order to ensure the control object match exactly the reference model, set the adjustable gain $k_{c}$ and two auxiliary signal generator $F_{1}$ and $F_{2}$ ' to make up the adaptive controller. The input of $F_{1}$ is the control volume $u$ of controlled object, There are $n-1$ adjustable parameters $c_{i}(i=1,2, \cdots, n-1)$. The input of $F_{2}$ is the output $y$ of controlled object, There are $n$ adjustable parameters $d_{i}(i=1,2, \cdots, n)$.Adding the feed-forward gain $k_{c}$, totally $2 n$ adjustable parameters. According to Narendra adaptive control law and adaptive controller can be designed as follows[6].

Structuring the auxiliary signal state equation: 


$$
\left\{\begin{array}{c}
\dot{\mathbf{v}}_{1}=\mathbf{A}_{f} \mathbf{v}_{1}+\mathbf{b}_{f} u \\
\dot{\mathbf{v}}_{2}=\mathbf{A}_{f} \mathbf{v}_{2}+\mathbf{b}_{f} y
\end{array}\right.
$$

$\mathbf{A}_{f}$ is the $\operatorname{proposed}(n-1) \times(n-1)$ asymptotically stable matrix, $\mathbf{b}_{f}=[00 \cdots 1]^{T} \in \mathbf{R}^{(n-1) \times 1}$.

Adjustable parameter adaptive law is as follows:

$$
\boldsymbol{\theta}(t)=\boldsymbol{\Gamma} \boldsymbol{\varphi}(t) e(t)
$$

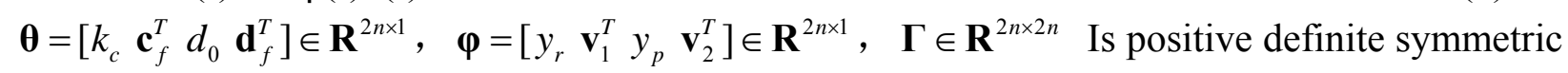
matrix..

The adaptive control law is as follows:

$$
u(t)=\boldsymbol{\theta}^{T}(t) \varphi(t)
$$

\section{Simulation}

Controlled object model for nonlinear system is

Reference model is

$$
y(k+1)=y(k) /\left(y(k)^{3}+2\right)+u(k)+3 u(k-1)
$$

$$
y(k+1)=0.7 y(k)+1.25 u(k)+0.75 u(k-1)
$$

System structure is unknown, the training sample structure of inverse model is as follows:

$$
\begin{aligned}
X(k) & =[y(k+3), y(k+2), y(k+1), u(k+1), u(k)] \\
Y(k) & =u(k+2)
\end{aligned}
$$

Kernel function is polynomial kernel function $K\left(\mathbf{x}, \mathbf{x}_{\mathbf{i}}\right)=\left(\mathbf{x}_{\mathbf{1}}^{\mathrm{T}} \mathbf{x}+t\right)^{d}, d=3, t=1.5$. The regularization parameter $C=1000$. Sampling time is $0.01 \mathrm{~s}$. The simulation time is $2 s$, System identification signal adopted sinusoidal signal $0.5 \sin (2 \pi t)+0.5$. The output of the LS_SVM inverse modeling and error are respectively as Fig. 3 and Fig.4. Modeling error is $10^{-3}$ orders of magnitude. It shows that the precision of nonlinear system based on LS_SVM inverse model is very high. The adaptive gain matrix $\Gamma=10 \mathbf{I}_{4 \times 4}$. The reference trajectory $r(t)$ is a square wave signal with 10 amplitude. Adopted the compound control method, the simulation results are shown in Fig.5

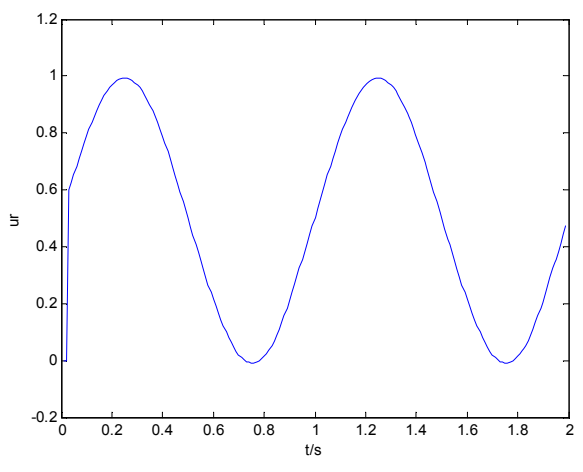

Fig.3 the output of sinusoidal signal

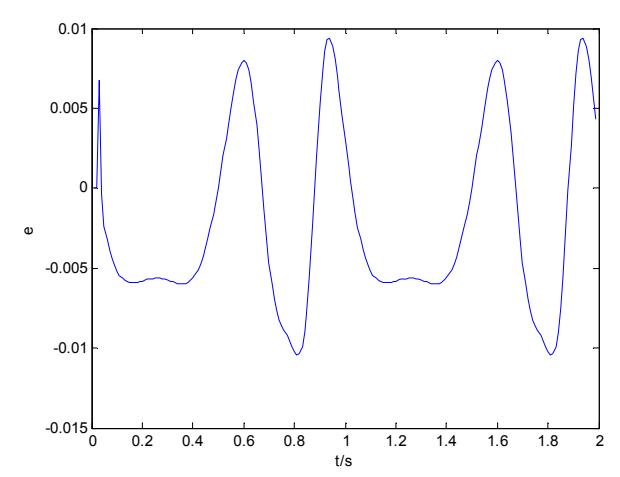

Fig.4 inverse modeling error of the sinusoidal signal 

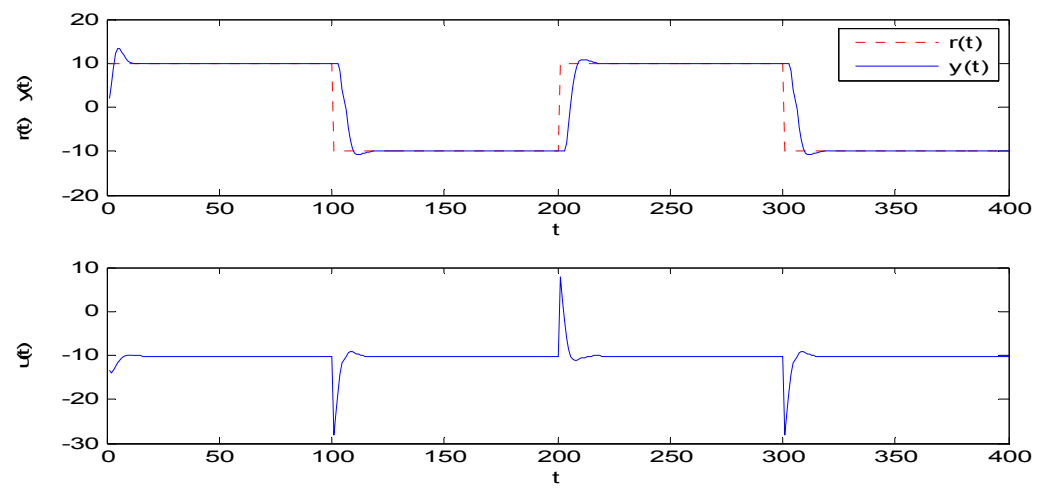

Fig. 5 the effect of compound control

\section{Conclusion}

As can be seen from the simulation results that established the inverse model of nonlinear system is very precise based on LS_SVM and could meet the needs of the inverse model control. The model reference adaptive control based on LS_SVM inverse model could effectively track on the expect output and get very satisfactory control performance

\section{Reference}

[1]HUANG yin-tong, ZHANG Shao-Me. A inverse control system based On least squares support vector machine and PID $[\mathrm{J}]$. Journal of Mechanical\&Electrical Engineering, 2010, 27(2): 75-78.

[2]Suykens J A K . Support vector machines : a nonlinear modeling and control perspective[J]. European Journal of Control, 2001, 7(2-3): 311-327.

[3]LI L J, SU H Y and CHU J. Generalized predictive control with online least squares support vector machines[J]. Acta Automatica Sinica, 2007, 33(1 J): 1182-1188.

[4] Liu Xiaojing, Yi Jianjiang, Zhao Dongbin, etc. The adaptive inverse disturbance elimination control system based on least squares support vector machine [J]. Control and decision, 2005, 20 (8): 947-950.

[5] Ou Yangjun, Wang Teng. The SVM application in the random vibration adaptive inverse control online [J]. Journal of applied mechanics, 2007, 24 (4):531-534.

[6] Liu Fucai,Liu Shuo. Model reference adaptive inverse control based on T - S fuzzy model [J]. Journal of systems engineering and electronics, 2013, 35 (9):1940-1947 